\title{
New Microscopic Connections of Thermodynamics
}

\author{
A. Plastino ${ }^{1}$ and M. Casas ${ }^{2}$ \\ ${ }^{1}$ Facultad de C. Exactas, Universidad Nacional de La Plata \\ IFLP-CONICET, C.C. 727, 1900 La Plata \\ ${ }^{2}$ Physics Departament and IFISC-CSIC, University of Balearic Islands \\ 07122 Palma de Mallorca \\ ${ }^{1}$ Argentina \\ ${ }^{2}$ Spain
}

\section{Introduction}

This is a work that discusses the foundations of statistical mechanics (SM) by revisiting its postulates in the case of the two main extant versions of the theory. A third one will here we added, motivated by the desire for an axiomatics that possesses some thermodynamic "flavor", which does not happen with neither of the two main SM current formulations, namely, those of Gibbs' $(1 ; 2)$, based on the ensemble notion, and of Jaynes', centered on MaxEnt $(3 ; 4 ; 5)$.

One has to mention at the outset that we "rationally understand" some physical problem when we are able to place it within the scope and context of a specific "Theory". In turn, we have a theory when we can both derive all the known interesting results and successfully predict new ones starting from a small set of axioms. Paradigmatic examples are von Neumann's axioms for Quantum Mechanics, Maxwell's equations for electromagnetism, Euclid's axioms for classical geometry, etc. $(1 ; 3)$.

Boltzmann's main goal in inventing statistical mechanics during the second half of the XIX century was to explain thermodynamics. However, he did not reach the axiomatic stage described above. The first successful SM theory was that of Gibbs (1902) (2), formulated on the basis of four ensemble-related postulates (1). The other great SM theory is that of Jaynes' (4), based upon the MaxEnt axiom (derived from Information Theory): ignorance is to be extremized (with suitable constraints).

Thermodynamics (TMD) itself has also been axiomatized, of course, using four macroscopic postulates (6). Now, the axioms of SM and of thermodynamics belong to different worlds altogether. The former speak of either "ensembles" (Gibbs), which are mental constructs, or of "observers' ignorance" (Jaynes), concepts germane to thermodynamics' language, that refers to laboratory-parlance. In point of fact, TMD enjoys a very particular status in the whole of science, as the one and only theory whose axioms are empirical statements (1).

Of course, there is nothing to object to the two standard SM-axiomatics referred to above. However, a natural question emerges: would it be possible to have a statistical mechanics derived from axioms that speak, as far as possible, the same language as that of thermodynamics? To what an extent is this feasible? It is our intention here that of attempting a serious discussion of such an issue and try to provide answers to the query, following ideas developed in $(7 ; 8 ; 9 ; 10 ; 11 ; 12 ; 13)$. 


\section{Thermodynamics' axioms}

Thermodynamics can be thought of as a formal logical structure whose axioms are empirical facts, which gives it a unique status among the scientific disciplines (1). The four postulates we state below are entirely equivalent to the celebrated three laws of thermodynamics (6):

1. For every system there exists a quantity $E$, called the internal energy, such that a unique $E$-value is associated to each of its states. The difference between such values for two different states in a closed system is equal to the work required to bring the system, while adiabatically enclosed, from one state to the other.

2. There exist particular states of a system, called the equilibrium ones, that are uniquely determined by $E$ and a set of extensive (macroscopic) parameters $A_{v}, v=1, \ldots, M$. The number and characteristics of the $A_{v}$ depends on the nature of the system (14).

3. For every system there exists a state function $S\left(E, \forall A_{v}\right)$ that (i) always grows if internal constraints are removed and (ii) is a monotonously (growing) function of $E$. $S$ remains constant in quasi-static adiabatic changes.

4. $S$ and the temperature $T=\left[\frac{\partial E}{\partial S}\right]_{A_{1}, \ldots, A_{M}}$ vanish for the state of minimum energy and are $\geq 0$ for all other states.

From the second and 3rd. Postulates we will extract and highlight the following two assertions, that are essential for our purposes

- Statement 3a) for every system there exists a state function $S$, a function of $E$ and the $A_{v}$

$$
S=S\left(E, A_{1}, \ldots, A_{M}\right) .
$$

- Statement $3 b) S$ is a monotonous (growing) function of $E$, so that one can interchange the roles of $E$ and $S$ in (1) and write

$$
E=E\left(S, A_{1}, \ldots, A_{M}\right),
$$

Eq. (2) clearly indicates that

$$
d E=\frac{\partial E}{\partial S} d S+\sum_{v} \frac{\partial E}{\partial A_{v}} d A_{v} \Rightarrow d E=T d S+\sum_{v} P_{v} d A_{v}
$$

with $P_{v}$ generalized pressures and the temperature $T$ defined as (6)

$$
T=\left(\frac{\partial E}{\partial S}\right)_{\left[\forall A_{v}\right]} .
$$

Eq. (3) will play a central role in our considerations, as discussed below.

If we know $S\left(E, A_{1}, \ldots, A_{n}\right)$ (or, equivalently because of monotonicity,

$\left.E\left(S, A_{1}, \ldots, A_{n}\right)\right)$ we have a complete thermodynamic description of a system. It is often experimentally more convenient to work with intensive variables.

Let define $S \equiv A_{0}$. The intensive variable associated to the extensive $A_{i}$, to be called $P_{i}$ is:

$$
\begin{gathered}
P_{0} \equiv T=\left[\frac{\partial E}{\partial S}\right]_{A_{1}, \ldots, A_{n}}, 1 / T=\beta \\
P_{j} \equiv \lambda_{j} / T=\left[\frac{\partial E}{\partial A_{j}}\right]_{S, A_{1}, \ldots, A_{j-1}, A_{j+1}, \ldots, A_{n}}
\end{gathered}
$$


Any one of the Legendre transforms that replaces any $s$ extensive variables by their associated intensive ones $(\beta, \lambda$ 's will be Lagrange multipliers in SM)

$$
L_{r_{1}, \ldots, r_{s}}=E-\sum_{j} P_{j} A_{j},\left(j=r_{1}, \ldots, r_{s}\right)
$$

contains the same information as either $S$ or $E$. The transform $L_{r_{1}, \ldots, r_{S}}$ is a function of $n-s$ extensive and $s$ intensive variables. This is called the Legendre invariant structure of thermodynamics.

\section{Gibbs' approach to statistical mechanics}

In 1903 Gibbs formulated the first axiomatic theory for statistical mechanics (1), that revolves around the basic physical concept of phase space. Gibbs calls the "phase of the system" to its phase space (PS) precise location, given by generalized coordinates and momenta. His postulates refer to the notion of ensemble (a mental picture), an extremely great collection of $N$ independent systems, all identical in nature with the one under scrutiny, but differing in phase. One imagines the original system to be repeated many times, each of them with a different arrangement of generalized coordinates and momenta. Liouville's celebrated theorem of volume conservation in phase space for Hamiltonian motion applies. The ensemble amounts to a distribution of N PS-points, representative of the "true" system. $N$ is so large that one can speak of a density $D$ at the PS-point $\phi=q_{1}, \ldots, q_{N} ; p_{1}, \ldots, p_{N}$, with $D=D\left(q_{1}, \ldots, q_{N} ; p_{1}, \ldots, p_{N}, t\right) \equiv D(\phi)$, with $t$ the time, and, if we agree to call $d \phi$ the pertinent volume element,

$$
N=\int d \phi D ; \forall \mathrm{t}
$$

If a system were to be extracted randomly from the ensemble, the probability of selecting one whose phase lies in a neighborhood of $\phi$ would be simply

$$
P(\phi)=D(\phi) / N \text {. }
$$

Consequently,

$$
\int P d \phi=1
$$

Liouville's theorem follows from the fact that, since phase-space points can not be "destroyed", if

$$
N_{12}=\int_{\phi_{1}}^{\phi_{2}} D d \phi
$$

then

$$
\frac{d N_{12}}{d t}=0
$$

An appropriate analytical manipulation involving Hamilton's canonical equations of motion then yields the theorem in the form (1)

$$
\dot{D}+\sum_{i}^{N} \frac{\partial D}{\partial p_{i}} \dot{p}_{i}+\sum_{i}^{N} \frac{\partial D}{\partial q_{i}} \dot{q}_{i}=0,
$$

entailing what Gibbs calls the conservation of density-in-phase. 
Equilibrium is simply the statement $\dot{D}=0$, i. e.,

$$
\sum_{i}^{N} \frac{\partial D}{\partial p_{i}} \dot{p}_{i}+\sum_{i}^{N} \frac{\partial D}{\partial q_{i}} \dot{q}_{i}=0 .
$$

\subsection{Gibbs' postulates for statistical mechanics}

The following statements wholly and thoroughly explain in microscopic fashion the corpus of equilibrium thermodynamics (1).

- The probability that at time $t$ the system will be found in the dynamical state characterized by $\phi$ equals the probability $P(\phi)$ that a system randomly selected from the ensemble shall possess the phase $\phi$ will be given by (6).

- All phase-space neighborhoods (cells) have the same a priori probability.

- $D$ depends only upon the system's Hamiltonian.

- The time-average of a dynamical quantity $F$ equals its average over the ensemble, evaluated using $D$.

\section{Information theory (IT)}

The IT-father, Claude Shannon, in his celebrated foundational paper (15), associates a degree of knowledge (or ignorance) to any normalized probability distribution $p(i),(i=1, \ldots, N)$, determined by a functional of the $\left\{p_{i}\right\}$ called the information measure $I\left[\left\{p_{i}\right\}\right]$, giving thus birth to a new branch of mathematics, that was later axiomatized by Kinchin (16), on the basis of four axioms, namely,

- $I$ is a function ONLY of the $p(i)$,

- $I$ is an absolute maximum for the uniform probability distribution,

- $I$ is not modified if an $N+1$ event of probability zero is added,

- Composition law.

\subsection{Composition}

Consider two sub-systems $\left[\Sigma^{1},\left\{p^{1}(i)\right\}\right]$ and $\left[\Sigma^{2},\left\{p^{2}(j)\right\}\right]$ of a composite system $[\Sigma,\{p(i, j)\}]$ with $p(i, j)=p^{1}(i) p^{2}(j)$. Assume further that the conditional probability distribution (PD) $Q(j \mid i)$ of realizing the event $j$ in system 2 for a fixed $i$-event in system 1 . To this PD one associates the information measure $I[Q]$. Clearly,

$$
p(i, j)=p^{1}(i) Q(j \mid i) .
$$

Then Kinchin's fourth axiom states that

$$
I(p)=I\left(p^{1}\right)+\sum_{i} p^{1}(i) I(Q(j \mid i)) .
$$

An important consequence is that, out of the four Kinchin axioms one finds that Shannons's measure

$$
S=-\sum_{i=1}^{N} p(i) \ln [p(i)],
$$

is the one and only measure complying with them. 


\section{Information theory and statistical mechanics}

Information theory (IT) entered physics via Jaynes' Maximum Entropy Principle (MaxEnt) in 1957 with two papers in which statistical mechanics was re-derived à la IT $(5 ; 17 ; 18)$, without appeal to Gibbs' ensemble ideas. Since IT's central concept is that of information measure (IM) $(5 ; 15 ; 17 ; 19)$, a proper understanding of its role must at the outset be put into its proper perspective.

In the study of Nature, scientific truth is established through the agreement between two independent instances that can neither bribe nor suborn each other: analysis (pure thought) and experiment (20). The analytic part employs mathematical tools and concepts. The following scheme thus ensues:

\section{WORLD OF MATHEMATICAL ENTITIES $\Leftrightarrow$ LABORATORY}

The mathematical realm was called by Plato Topos Uranus (TP). Science in general, and physics in particular, is thus primarily (although not exclusively, of course) to be regarded as a TP $\Leftrightarrow$ "Experiment" two-way bridge, in which TP concepts are related to each other in the form of "laws" that are able to adequately describe the relationships obtaining among suitable chosen variables that describe the phenomenon one is interested in. In many instances, although not in all of them, these laws are integrated into a comprehensive theory (e.g., classical electromagnetism, based upon Maxwell's equations) $(1 ; 21 ; 22 ; 23 ; 24)$.

If recourse is made to MaxEnt ideas in order to describe thermodynamics, the above scheme becomes now:

IT as a part of $\mathrm{TP} \Leftrightarrow$ Thermal Experiment,

or in a more general scenario:

IT $\Leftrightarrow$ Phenomenon to be described.

It should then be clear that the relation between an information measure and entropy is:

$\mathrm{IM} \Leftrightarrow$ Entropy $S$.

One can then state that an IM is not necessarily an entropy! How could it be? The first belongs to the Topos Uranus, because it is a mathematical concept. The second to the laboratory, because it is a measurable physical quantity. All one can say is that, at most, in some special cases, an association $I M \Leftrightarrow$ entropy $S$ can be made. As shown by Jaynes (5), this association is both useful and proper in very many situations.

\section{MaxEnt rationale}

The central IM idea is that of giving quantitative form to the everyday concept of ignorance (17). If, in a given scenario, $N$ distinct outcomes $(i=1, \ldots, N)$ are possible, then three situations may ensue (17):

1. Zero ignorance: predict with certainty the actual outcome.

2. Maximum ignorance: Nothing can be said in advance. The $N$ outcomes are equally likely.

3. Partial ignorance: we are given the probability distribution $\left\{P_{i}\right\} ; i=1, \ldots, N$.

The underlying philosophy of the application of IT ideas to physics via the celebrated Maximum Entropy Principle (MaxEnt) of Jaynes' (4) is that originated by Bernoulli and 
Laplace (the fathers of Probability Theory) (5), namely: the concept of probability refers to an state of knowledge. An information measure quantifies the information (or ignorance) content of a probability distribution (5). If our state of knowledge is appropriately represented by a set of, say, $M$ expectation values, then the "best", least unbiased probability distribution is the one that

- reflects just what we know, without "inventing" unavailable pieces of knowledge $(5 ; 17)$ and, additionally,

- maximizes ignorance: the truth, all the truth, nothing but the truth.

Such is the MaxEnt rationale (17). It should be then patently clear that, in using MaxEnt, one is NOT maximizing a physical entropy. One is maximizing ignorance in order to obtain the least biased distribution compatible with the a priori knowledge.

\subsection{Jaynes mathematical formulation}

As stated above, Statistical Mechanics and thereby Thermodynamics can be formulated on the basis of Information Theory if the statistical operator $\hat{\rho}$ is obtained by recourse to the MAXIMUM ENTROPY PRINCIPLE (MaxEnt). Consequently, we have the MaxEnt principle: MaxEnt: Assume your prior knowledge about the system is given by the values of $M$ expectation values $<A_{1}>, \ldots,<A_{M}>$. Then $\hat{\rho}$ is uniquely fixed by extremizing $I(\hat{\rho})$ subject to the constraints given by the $M$ conditions

$$
<A_{j}>=\operatorname{Tr}\left[\hat{\rho} \hat{A}_{j}\right]
$$

(entailing the introduction of $M$ associated Lagrange multipliers $\lambda_{i}$ ) plus normalization of $\hat{\rho}$ (entailing a normalization Lagrange multiplier $\xi$.) In the process one discovers that $I \equiv S$, the equilibrium Boltzmann's entropy, if our prior knowledge $\left.\left\langle A_{1}\right\rangle, \ldots,<A_{M}\right\rangle$ refers to extensive quantities. Such $I$-value, once determined, yields complete thermodynamical information with respect to the system of interest.

\section{Possible new axioms for SM}

Both Gibbs' and MaxEnt are beautiful, elegant theories that satisfactorily account for equilibrium thermodynamics. Whys should we be looking for still another axiomatics? Precisely because, following Jaynes IT-spirit, one should be endeavoring to use all information actually available to us in building up our theoretic foundations, and this is not done in MaxEnt, as we are about to explicitate.

Our main argument revolves around the possibility of giving Eq. (3), an empirical statement, the status of an axiom, actually employing thus a piece of information available to us without any doubt. This constitutes the first step in our present discourse. More explicitly, in order to concoct a new SM-axiomatics, we start by establishing as a theoretic postulate the following macroscopic assertion:

Axiom (1)

$$
d E=T d S+\sum_{v} P_{v} d A_{v}
$$

Since this is a macroscopic postulate in a microscopic axiomatics' corpus, it is pertinent now to ask ourselves which is the minimum amount of microscopic information that we would have to add to such an axiomatics in order to get all the microscopic results of equilibrium statistical mechanics. Since we know about Kinchin's postulates, we borrow from him his 
first one. Consequently, we conjecture at this point, and will prove below, that the following statements meets the bill:

Axiom (2) If there are $\mathcal{N}$ microscopic accessible states labelled by $i$, of microscopic probability $p_{i}$, then

$$
S=S\left(p_{1}, p_{2}, \ldots, p_{\mathcal{N}}\right)
$$

In what follows, the number of microstates will also be denoted by $W$.

Now, we will take as a postulate something that we actually know form both quantum and classical mechanics.

Axiom (3) The internal energy $E$ and the external parameters $A_{v}$ are to be regarded as expectation values of suitable operators, respectively the hamiltonian $H$ and $\mathcal{R}_{v}$ (i.e., $A_{v} \equiv<\mathcal{R}_{v}>$ ).

Thus the $A_{v}$ (and also E) will depend on the eigenvalues of these operators and on the probability set. (The energy eigenvalues depend of course upon the $\mathcal{R}_{v}$.) The reader will immediately realize that Axiom (2) is just a form of Boltzmann's "atomic" conjecture, pure and simple. In other words, macroscopic quantities are statistical averages evaluated using a microscopic probability distribution (25). It is important to realize that our three new axioms are statements of fact in the sense that they are borrowed either from experiment or from pre-existent theories. In fact, the 3 axioms do not incorporate any knew knowledge at all! In order to prove that our above three postulates do allow one to build up the mighty SM-edifice we will show below that they are equivalent to Jaynes' SM-axiomatics (4).

Of course, the main SM-goal is that of ascertaining which is the PD (or the density operator) that best describes the system of interest. Jaynes appeals in this respect to his MaxEnt postulate, the only one needed in this SM-formulation. We restate it below for the sake of fixing notation.

MaxEnt axiom: assume your prior knowledge about the system is given by the values of $\mathrm{M}$ expectation values

$$
A_{1} \equiv<\mathcal{R}_{1}>, \ldots, A_{R} \equiv<\mathcal{R}_{M}>.
$$

Then, $\rho$ is uniquely fixed by extremizing the information measure $I(\rho)$ subject to $\rho$-normalization plus the constraints given by the $M$ conditions constituting our assumed foreknowledge

$$
A_{v}=<\mathcal{R}_{v}>=\operatorname{Tr}\left[\rho \mathcal{R}_{v}\right]
$$

This leads, after a Lagrange-constrained extremizing process, to the introduction of $M$ Lagrange multipliers $\lambda_{v}$, that one assimilates to the generalized pressures $P_{v}$. The truth, the whole truth, nothing but the truth (17). If the entropic measure that reflects our ignorance were not maximized, we would be inventing information that we do not actually possess. In performing the variational process Jaynes discovers that, provided one multiplies the right-hand-side of the information measure expression by Boltzmann's constant $k_{B}$, the IM equals the entropic one. Thus, $I \equiv S$, the equilibrium thermodynamic entropy, with the caveat that our prior knowledge $A_{1}=\left\langle\mathcal{R}_{1}\right\rangle, \ldots, A_{M}=\left\langle\mathcal{R}_{M}>\right.$ must refer just to extensive quantities. Once $\rho$ is at hand, $I(\rho)$ yields complete microscopic information with respect to the system of interest. Our goal should be clear now. We need to prove that our new axiomatics, encapsulated by (15) and (16), is equivalent to MaxEnt. 


\section{Equivalence between MaxEnt and our new axiomatics}

We will here deal with the classical instance only. The quantal extension is of a straightforward nature. Consider a generic change $p_{i} \rightarrow p_{i}+d p_{i}$ constrained by Eq. (15), that is, the change $d p_{i}$ must be of such nature that (15) is verified. Obviously, $S, A_{j}$, and $E$ will change with $d p_{i}$ and, let us insist, these changes are constrained by (15). We will not specify the information measure, as several possibilities exist (26). For a detailed discussion of this issue see (27). In this endeavor our ingredients are

- an arbitrary, smooth function $f(p)$ that allows us to express the information measure in the fashion

$$
I \equiv S\left(\left\{p_{i}\right\}\right)=\sum_{i} p_{i} f\left(p_{i}\right),
$$

such that $S\left(\left\{p_{i}\right\}\right)$ is a concave function,

- $M$ quantities $A_{v}$ that represent mean values of extensive physical quantities $\left\langle\mathcal{R}_{v}\right\rangle$, that take, for the micro-state $i$, the value $a_{i}^{v}$ with probability $p_{i}$,

- another arbitrary smooth, monotonic function $g\left(p_{i}\right)(g(0)=0 ; g(1)=1)$. It is in order to use generalized, non-Shannonian entropies that we have slightly generalized mean-value definitions using the function $g$.

We deal then with (we take $A_{1} \equiv E$ ), using the function $g$ to evaluate (generalized) expectation values,

$$
\begin{gathered}
A_{v} \equiv\left\langle\mathcal{R}_{v}\right\rangle=\sum_{i}^{W} a_{i}^{v} g\left(p_{i}\right) ; v=2, \ldots, M, \\
E=\sum_{i}^{W} \epsilon_{i} g\left(p_{i}\right),
\end{gathered}
$$

where $\epsilon_{i}$ is the energy associated to the microstate $i$. The probability variations $d p_{i}$ will now generate corresponding changes $d S, d A_{v}$, and $d E$ in, respectively, $S$, the $A_{v}$, and $E$.

\subsection{Proof, part I}

The essential point of our present methodology is to enforce obedience to

$$
d E-T d S+\sum_{v=1}^{W} d A_{v} \lambda_{v}=0
$$

with $T$ the temperature and $\lambda_{v}$ generalized pressures. We use now the expressions (19), (20), and (21) so as to cast (22) in terms of the probabilities, according to an infinitesimal probabilities' change

$$
p_{i} \rightarrow p_{i}+d p_{i}
$$

If we expand the resulting equation up to first order in the $d p_{i}$, it is immediately found, after a little algebra, that the following set of equations ensues $(7 ; 8 ; 9 ; 10 ; 11 ; 12 ; 13)$ (remember that the Lagrange multipliers $\lambda_{v}$ are identical to the generalized pressures $P_{v}$ of Eq. (3))

$$
\begin{gathered}
C_{i}^{(1)}=\left[\sum_{v=1}^{M} \lambda_{v} a_{i}^{v}+\epsilon_{i}\right] \\
C_{i}^{(2)}=-T \frac{\partial S}{\partial p_{i}}
\end{gathered}
$$




$$
\sum_{i}\left[C_{i}^{(1)}+C_{i}^{(2)}\right] d p_{i} \equiv \sum_{i} K_{i} d p_{i}=0 .
$$

We can rearrange matters in the fashion

$$
\begin{gathered}
T_{i}^{(1)}=f\left(p_{i}\right)+p_{i} f^{\prime}\left(p_{i}\right) \\
T_{i}^{(2)}=-\beta\left[\left(\sum_{v=1}^{M} \lambda_{v} a_{i}^{v}+\epsilon_{i}\right) g^{\prime}\left(p_{i}\right)-K\right], \\
(\beta \equiv 1 / k T),
\end{gathered}
$$

so that we can recast (24) as

$$
T_{i}^{(1)}+T_{i}^{(2)}=0 ;(\text { for any } i),
$$

a relation whose importance will become manifest in Appendix I.

We wish that Eqs. (24) or (26) should yield one and just one $p_{i}$-expression, which it indeed does $(7 ; 8 ; 9 ; 10 ; 11 ; 12 ; 13)$. We do not need here, however, for our demonstration, an explicit expression for this probability distribution, as will be immediately realized below.

\subsection{Proof, part II: follow Jaynes' procedure}

Alternatively, proceed à la MaxEnt. This requires extremizing the entropy $S$ subject to the usual constraints in $E, A_{v}$, and normalization. The ensuing, easy to carry out Jaynes' variational treatment, can be consulted in $(7 ; 8 ; 9 ; 10 ; 11 ; 12 ; 13)$, that is (we set $\lambda_{1} \equiv \beta=1 / T$ )

$$
\delta_{p_{i}}\left[S-\beta\langle H\rangle-\sum_{v=2}^{M} \lambda_{v}\left\langle\mathcal{R}_{v}\right\rangle-\xi \sum_{i} p_{i}\right]=0,
$$

(we need also a normalization Lagrange multiplier $\xi$ ) is easily seen to yield as a solution the very set of Eqs. (24) as well! (see Appendix I for the proof). These equations arise then out of two clearly separate treatments: (I) our methodology, based on Eqs. (15) and (16), and (II), following the MaxEnt prescriptions. This entails that MaxEnt and our axiomatics co-imply each other, becoming thus equivalent ways of building up statistical mechanics. An important point is to be here emphasized with respect to the functional $S$-form.

The specific form of $S\left[p_{i}\right]$ is not needed neither in Eqs. (24) nor in (27)!

\section{What does all of this mean?}

We have already formally proved that our axiomatics is equivalent to MaxEnt, and serves thus as a foundation for equilibrium statistical mechanics. We wish now to dwell in deeper fashion into the meaning of our new SM-formulation. First of al it is to be emphasized that, in contrast to both Gibbs' and Jaynes' postlates, ours have zero new informational content, since they are borrowed either from experiment or from pre-existing theories, namely, information theory and quantum mechanics. In particular, we wish to dwell to a larger extent on both the informational and physical contents of our all-important Eqs. (24) or (26).

The first and second laws of thermodynamics are two of physics' most important statements. They constitute strong pillars of our present understanding of Nature. Of course, statistical mechanics (SM) adds an underlying microscopic substratum that is able to explain not only these two laws but the whole of thermodynamics itself $(6 ; 17 ; 28 ; 29 ; 30 ; 31)$. One of SM's basic ingredients is a microscopic probability distribution (PD) that controls the population 
of microstates of the system under consideration (28). Since we were here restricting our considerations to equilibrium situations, what we have been really doing here was to mainly concern ourselves with obtaining a detailed picture, from a new perspective $(7 ; 8 ; 9 ; 10 ; 11 ; 12$; 13), of how changes in the independent external parameters - thermodynamic parameters affect this micro-state population and, consequently, the entropy and the internal energy, i.e.,

reversible changes in external parameters $\Delta_{\text {param }} \rightarrow$ changes in the microscopic probability distribution $\rightarrow$ entropic $(d S)$ and internal energy $(d U)$ changes.

We regarded as independent external parameters both extensive and intensive quantities defining the macroscopic thermodynamic state of the system. It is well-known that the extensive parameters, always known with some (experimental) uncertainty, help to define the Hilbert space (HS) in which the system can be represented. The intensive parameters are associated with some physical quantities of which only the average value is known. They are related to the mean values of operators acting on the HS previously defined. The eigenvalues of these operators are, therefore, functions of the extensive parameters defining the HS. The microscopic equilibrium probability distribution (PD) is an explicit function of the intensive parameters and an implicit function - via the eigenvalues of the above referred to operators (known in average) - of the extensive parameters defining the HS.

What is the hard core of the new view-point of $(7 ; 8 ; 9 ; 10 ; 11 ; 12 ; 13)$ ? It consists, as will be detailed below, in

- enforcing the relation $d U=T d S+\sum_{v} P_{v} d A_{v}$ in an infinitesimal microscopic change $p_{i} \rightarrow$ $p_{i}+d p_{i}$ of the probability distribution (PD) that describes the equilibrium properties of an arbitrary system and ascertaining that

- this univocally determines the PD, and furthermore,

- that the ensuing $\left\{p_{i}\right\}$ coincides with that obtained following the maximum entropy principle (MaxEnt) tenet of extremizing the entropy $S$ subject to an assumedly known mean value $U$ of the system's energy.

Consider now only infinitesimal macroscopic parameter-changes (as opposite to the microscopic PD-ones dealt with in (7)), according to the scheme below.

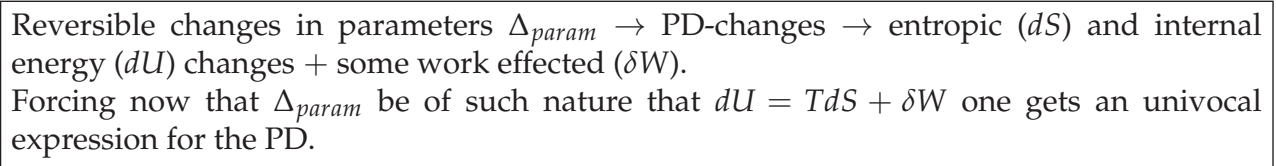

That is, we study variations in both the (i) intensive and (ii) extensive parameters of the system and wish to ascertain just how these variations materialize themselves into concrete thermal relations.

\subsection{Homogeneous, isotropic, one-component systems}

For simplicity, consider just simple, one-component systems (6) composed by a single chemical species, macroscopically homogeneous, and isotropic (6). The macroscopic equilibrium thermal state of such a simple, one-component system is described, in self-explanatory notation, by $T, V, N(6)$. Focus attention upon a quite general information measure $S$ that, according to Kinchin's axioms for information theory depends exclusively on of the probability distribution $\left\{p_{i}\right\}$. We use again the specific but rather general form given above 
for $S$, viz.,

$$
S=k \sum_{i=1}^{W} p_{i} f\left(p_{i}\right)
$$

with $W$ the number of microscopic states, $k=$ Boltzmann's constant, and the sum running over a set of quantum numbers, collectively denoted by $i$ (characterizing levels of energy $\epsilon_{i}$ ), that specify an appropriate basis in Hilbert's space ( $f$ is an arbitrary smooth function of the $p_{i}$ such that $p f(p)$ is concave).

Remember that the quantity $U$ represents the mean value of the Hamiltonian, and, as befits an homogeneous, isotropic, one-component system in the Helmholtz free energy representation (6) we have

1. as external parameter the volume $(V)$ and the number of particles $(N)$ ("exactly" known and used to define the Hilbert space),

2. as intensive variable the temperature $T$, associated with the mean value $U$ of the internal energy $E$, i.e., $U=\langle E\rangle$.

The energy eigenvalues of the Hamiltonian $\epsilon_{i}$ are, obviously, functions of the volume and of the number of particles, namely, $\left\{\epsilon_{i}\right\}=\left\{\epsilon_{i}(V, N)\right\}$. From now on, for simplicity, we take $N$ as fixed, and drop thereby the dependence of the energy eigenvalues on $N$, i.e., $\left\{\epsilon_{i}\right\}=\left\{\epsilon_{i}(V)\right\}$. The probability distribution (PD) depends, then, on the external parameters in the fashion

$$
p_{i}=p_{i}\left(T, \epsilon_{i}(V)\right) \text {. }
$$

Remind that the mean energy $U=\langle E\rangle$ is given by

$$
U=\langle E\rangle=\sum_{i=1}^{W} g\left(p_{i}\right) \epsilon_{i} .
$$

The critical difference between what we attempt to do now and what was related above [Cf. Eq. (23)] is to be found in the following assumption, on which we entirely base our considerations in this Section:

the temperature $T$ and the volume $V$ reversibly change in the fashion

$$
T \rightarrow T+d T \text { and } V \rightarrow V+d V .
$$

As a consequence of (31), corresponding changes $d p_{i}, d S, d \epsilon_{i}$, and $d U$ are generated in, respectively, $p_{i}, S, \epsilon_{i}$, and $U$. Variations in, respectively, $p_{i}, S$, and $U$ write

$$
\begin{gathered}
d p_{i}=\frac{\partial p_{i}}{\partial T} d T+\sum_{j=1}^{W} \frac{\partial p_{i}}{\partial \epsilon_{j}} \frac{\partial \epsilon_{j}}{\partial V} d V \\
d S=\sum_{i=1}^{W} \frac{\partial S}{\partial p_{i}} \frac{\partial p_{i}}{\partial T} d T+\sum_{i, j=1}^{W} \frac{\partial S}{\partial p_{i}} \frac{\partial p_{i}}{\partial \epsilon_{j}} \frac{\partial \epsilon_{j}}{\partial V} d V,
\end{gathered}
$$

and, last but not least,

$$
d U=\sum_{i=1}^{W} \frac{\partial g}{\partial p_{i}} \frac{\partial p_{i}}{\partial T} \epsilon_{i} d T+\sum_{i, j=1}^{W} \frac{\partial g}{\partial p_{i}} \frac{\partial p_{i}}{\partial \epsilon_{j}} \frac{\partial \epsilon_{j}}{\partial V} \epsilon_{i} d V+\sum_{i=1}^{W} g\left(p_{i}\right) \frac{\partial \epsilon_{i}}{\partial V} d V,
$$


where, for simplicity, we have considered non-degenerate levels. Clearly, on account of normalization, the changes in $p_{i}$ must satisfy the relation

$$
\sum_{i} d p_{i}=0
$$

Note that if we deal with three thermodynamic parameters and one equation of state we can completely describe our system with any two of them (32). Here, we are choosing, as the two independent thermodynamic parameters, $T$ and $V$. It is important to remark that independent thermodynamic parameters do not mean natural parameters. For example, if $T$ and $V$ are now the independent thermodynamic parameters, the internal energy can be written as function of these parameters, i.e., $U(T, V)$. Clearly, $T$ and $V$ are not the natural parameters of the internal energy. These are $S$ and $V$. However, our developments require only independent parameters, that are not necessarily the natural ones (32).

\subsection{Macroscopic considerations}

Thermodynamics states that, in the present scenario, for a reversible process one has

$$
d U=\delta Q+\delta W=T d S+\delta W,
$$

where we have used the Clausius relation $\delta Q=T d S$. Multiplying Eq. (33) by $T$ we can recast Eq. (36) in the microscopic fashion (involving the microstates' PD)

$$
d U=T\left(\sum_{i=1}^{W} \frac{\partial S}{\partial p_{i}} \frac{\partial p_{i}}{\partial T} d T+\sum_{i, j=1}^{W} \frac{\partial S}{\partial p_{i}} \frac{\partial p_{i}}{\partial \epsilon_{j}} \frac{\partial \epsilon_{j}}{\partial V} d V\right)+\delta W
$$

which is to be compared with (34).

\subsection{Changes in the temperature}

Eqs. (34) and (37) must be equal for arbitrary changes in $T$ and $V$. We take this equality as the basis of our future considerations. As $T$ and $V$ can be changed in an independent way, let us first consider just changes in T. Enforcing equality in the coefficients of $d T$ appearing in Eqs. (34) and (37) we obtain (we are assuming, as it is obvious, that the mechanical $\delta W$ does not depend on the temperature)

$$
\sum_{i=1}^{W} \frac{\partial g}{\partial p_{i}} \frac{\partial p_{i}}{\partial T} \epsilon_{i} d T=T \sum_{i=1}^{W} \frac{\partial S}{\partial p_{i}} \frac{\partial p_{i}}{\partial T} d T,
$$

that must be satisfied together with [Cf. (32)]

$$
\sum_{i} d p_{i}=\sum_{i} \frac{\partial p_{i}}{\partial T} d T=0 .
$$

We recast now (38) in the fashion 


$$
\sum_{i=1}^{W}\left(\frac{\partial g}{\partial p_{i}} \epsilon_{i}-T \frac{\partial S}{\partial p_{i}}\right) \frac{\partial p_{i}}{\partial T} d T \equiv \sum_{i} K_{i} \frac{\partial p_{i}}{\partial T} d T=0 .
$$

Since the $W p_{i}$ 's are not independent $\left(\sum_{i=1}^{W} p_{i}=1\right)$, we can separate the sum in (40) into two parts, i.e.,

$$
\sum_{i=1}^{W-1}\left(\frac{\partial g}{\partial p_{i}} \epsilon_{i}-T \frac{\partial S}{\partial p_{i}}\right) \frac{\partial p_{i}}{\partial T} d T+\left(\frac{\partial g}{\partial p_{W}} \epsilon_{W}-T \frac{\partial S}{\partial p_{W}}\right) \frac{\partial p_{W}}{\partial T} d T=0
$$

Picking out level $W$ for special attention is arbitrary. Any other $i-$ level could have been chosen as well, as the example given below will illustrate. Taking into account now that, from Eq. (39),

$$
\frac{\partial p_{W}}{\partial T}=-\sum_{i=1}^{W-1} \frac{\partial p_{i}}{\partial T}
$$

we see that Eq. (41) can be rewritten as

$$
\sum_{i=1}^{W-1}\left[\left(\frac{\partial g}{\partial p_{i}} \epsilon_{i}-T \frac{\partial S}{\partial p_{i}}\right)-\left(\frac{\partial g}{\partial p_{W}} \epsilon_{W}-T \frac{\partial S}{\partial p_{W}}\right)\right] \frac{\partial p_{i}}{\partial T} d T=0 .
$$

As the $W-1 p_{i}$ 's are now independent, the term into brackets should vanish, which entails

$$
\frac{\partial g}{\partial p_{i}} \epsilon_{i}-T \frac{\partial S}{\partial p_{i}}-\left(\frac{\partial g}{\partial p_{W}} \epsilon_{W}-T \frac{\partial S}{\partial p_{W}}\right)=0,
$$

for all $i=1, \cdots, W-1$. Let us call the term into parentheses as

$$
K_{W}=\frac{\partial g}{\partial p_{W}} \epsilon_{W}-T \frac{\partial S}{\partial p_{W}} \equiv K=\text { constant. }
$$

Finally, we cast Eqs. (44) and (45) as

$$
\frac{\partial g}{\partial p_{i}} \epsilon_{i}-T \frac{\partial S}{\partial p_{i}}-K=0 ;(i=1, \cdots, W),
$$

an equation that we have encountered before [Cf. Eq. (24) with $g(x) \equiv x$ ] and that should yield a definite expression for any of the $W p_{i}{ }^{\prime}$ s. We did not care above about such an expression, but we do now. 
Example 1 Consider the Shannon orthodox instance

$$
\begin{aligned}
S & =-k \sum_{i} p_{i} \ln p_{i} \\
g\left(p_{i}\right) & =p_{i} \\
\partial S / \partial p_{i} & \left.=-k\left[\ln p_{i}+1\right]=k\left[\beta \epsilon_{i}+\ln Z-1\right]\right] .
\end{aligned}
$$

Here equation (46) yields the well known MaxEnt (and also Gibbs?) result

$$
\begin{aligned}
\ln p_{i} & =-\left[\beta \epsilon_{i}+\ln Z\right] ; \text { i.e., } \\
p_{i} & =Z^{-1} e^{-\epsilon_{i} / k T} \\
\ln Z & =1-K / k T, \text { and, finally, } \\
& \partial S / \partial p_{i}=k \beta\left(\epsilon_{i}-K\right), \\
\frac{\partial \ln Z}{\partial \epsilon_{i}}=-\beta p_{i} ; \frac{\partial p_{i}}{\partial \epsilon_{j}}= & -\beta p_{i}\left(\delta_{i j}-p_{j}\right) ; T \frac{\partial S}{\partial p_{i}} \frac{\partial p_{i}}{\partial \epsilon_{i}}=-\beta\left(\epsilon_{i}-K\right) p_{i},
\end{aligned}
$$

showing, as anticipated, that we could have selected any $i$-level among the $W$-ones without affecting the final result.

Thus, changes $\delta \beta$ in the inverse temperature $\beta$ completely specify the microscopic probability density $\left\{p_{\text {MaxEnt }}\right\}$ if they are constrained to obey the relation $d U=T d S+\delta W$, for any reasonable choice of the information measure $S$. This equivalence, however, can not be established in similar fashion if the extensive variable $V$ also changes. This is our next topic.

\subsection{Changes in the extensive parameter}

Let us now deal with the effect of changes in the extensive parameters that define the Hilbert space in which our system "lives" and notice that Eq. (37) can be written in the fashion $d U=$ $\delta Q+\delta W=T d S+\delta W \Rightarrow$

$$
d U=T\left(d T \sum_{i=1}^{W} \frac{\partial S}{\partial p_{i}} \frac{\partial p_{i}}{\partial T}+d V \sum_{i, j=1}^{W} \frac{\partial S}{\partial p_{i}} \frac{\partial p_{i}}{\partial \epsilon_{j}} \frac{\partial \epsilon_{j}}{\partial V}\right)+\delta W .
$$

That is, there are two ingredients entering $T d S$, namely,

$$
T d S=Q_{T} d T+Q_{V} d V ; \text { with } Q_{T}=T \sum_{i=1}^{W} \frac{\partial S}{\partial p_{i}} \frac{\partial p_{i}}{\partial T} .
$$

Our interest now lies in the second term. What is $Q_{V}$ ? Clearly we have 


$$
Q_{V}=T \sum_{i, j=1}^{W} \frac{\partial S}{\partial p_{i}} \frac{\partial p_{i}}{\partial \epsilon_{j}} \frac{\partial \epsilon_{j}}{\partial V} .
$$

Next, substitute the expression for $\left(\partial g / \partial p_{i}\right) \epsilon_{i}$ given by Eqs. (45) and (46),

$$
\frac{\partial g}{\partial p_{i}} \epsilon_{i}=T \frac{\partial S}{\partial p_{i}}+K ;(i=1, \ldots, W),
$$

into the second term of the R.H.S. of Eq. (34),

$$
\begin{aligned}
\sum_{i, j=1}^{W} \frac{\partial g}{\partial p_{i}} \frac{\partial p_{i}}{\partial \epsilon_{j}} \frac{\partial \epsilon_{j}}{\partial V} \epsilon_{i} d V & =\sum_{i, j=1}^{W}\left[T \frac{\partial S}{\partial p_{i}}+K\right] \frac{\partial p_{i}}{\partial \epsilon_{j}} \frac{\partial \epsilon_{j}}{\partial V} d V \\
& =T \sum_{i, j=1}^{W} \frac{\partial S}{\partial p_{i}} \frac{\partial p_{i}}{\partial \epsilon_{j}} \frac{\partial \epsilon_{j}}{\partial V} d V+K \sum_{i, j=1}^{W} \frac{\partial p_{i}}{\partial \epsilon_{j}} \frac{\partial \epsilon_{j}}{\partial V} d V \\
& =\left(T \sum_{i, j=1}^{W} \frac{\partial S}{\partial p_{i}} \frac{\partial p_{i}}{\partial \epsilon_{j}} \frac{\partial \epsilon_{j}}{\partial V}\right) d V=Q_{V} d V
\end{aligned}
$$

on account of the fact that

$$
K \sum_{i, j=1}^{W} \frac{\partial p_{i}}{\partial \epsilon_{j}} \frac{\partial \epsilon_{j}}{\partial V} d V=0 ; \text { since }(\partial / \partial V) \sum_{i} p_{i}=0
$$

We recognize in the term $Q_{V} d V$ of the last line of (54) the microscopic interpretation of a rather unfamiliar "volume contribution" to Clausius' relation $\delta Q=T d S$ (dQ-equations (32)). Notice that we are not explicitly speaking here of phase-changes. We deal with reversible processes. If the change in volume were produced by a phase-change one would reasonably be tempted to call the term $Q_{V} d V$ a "latent" heat.

Thus, associated with a change of state in which the volume is modified, we find in the term $Q_{V} d V$ the microscopic expression of a "heat" contribution for that transformation, i.e., the heat given up or absorbed during it. It we wish to call it "latent", the reason would be that it is not associated with a change in temperature. Thus, we saw just how changes in the equilibrium PD caused by modifications in the extensive parameter defining the Hilbert space of the system give also a contribution to the "heat part" of the $d U=T d S+\delta W$ relation. 
Example 2: In the Shannon instance discussed in Example 1 one has [Cf. (48) and (49)]

$$
\begin{gathered}
\frac{\partial p_{i}}{\partial \epsilon_{i}}=-\beta p_{i}\left(1-Z^{-1}\right), \\
T \frac{\partial S}{\partial p_{i}} \frac{\partial p_{i}}{\partial \epsilon_{i}}=-\beta\left(\epsilon_{i}-K\right) p_{i}, \\
Q_{V}=-\sum_{i} \beta\left(\epsilon_{i}-K\right) p_{i} \frac{\partial \epsilon_{i}}{\partial V}\left(1-Z^{-1}\right) .
\end{gathered}
$$

Since the origin of the energy scale is arbitrary, in summing over $i$ we can omit the $K$-term by changing the energy-origin and one may write

$$
Q_{V}=-\sum_{i} \beta \epsilon_{i} p_{i} \frac{\partial \epsilon_{i}}{\partial V}\left(1-Z^{-1}\right)
$$

Foe a particle of mass $m$ in an ideal gas ( $N$ particles) the energy $\epsilon_{i}$ is given by (29)

$$
\begin{gathered}
\epsilon_{i}=\tau V^{-2 / 3} \vec{n}_{i}^{2} ; \tau=\frac{(\pi \hbar)^{2}}{2 m} ; \vec{n}_{i}^{2} \equiv\left(n_{x}^{2}, n_{y}^{2}, n_{z}^{2}\right) \\
n_{x}, n_{y}, n_{z} \text { a set of three integers } \\
\frac{\partial \epsilon_{i}}{\partial V}=-(2 / 3) \epsilon_{i} / V
\end{gathered}
$$

Thus, the microscopic expression for $Q_{V}$ turns out to be

$$
Q_{V}=(2 \beta / 3 V)\left\langle E^{2}\right\rangle\left(1-Z^{-1}\right),
$$

which indeed has dimension of (energy/volume).

Finally, for Eq. (34) to become equal to Eq. (50) we have to demand, in view of the above developments,

$$
\delta W=d V\left[\sum_{i} g\left(p_{i}\right) \frac{\partial \epsilon_{i}}{\partial V}\right],
$$

the quantity within the brackets being the mean value,

$$
\left\langle\frac{\partial E}{\partial V}\right\rangle=\sum_{i} g\left(p_{i}\right) \frac{\partial \epsilon_{i}}{\partial V},
$$

usually associated in the textbooks with the work done by the system.

Summing up, our analysis of simple systems in the present Section has shown that

- by considering changes $d T$ and $d V$ and how they influence the microscopic probability distribution if these variations are forced to comply with the relation (36) $d U=T d S+\delta W$ we ascertain that

- changes in the intensive parameter give contributions only related to heat and lead to the attaining the equilibrium PD (an alternative way to the MaxEnt principle) and

- changes in the extensive-Hilbert-space-determining parameter lead to two contributions

1. one related to heat and

2. the other related to work. 


\section{Other entropic forms}

We illustrate now our procedure with reference to information measures not of the Shannon logarithmic form. We use mostly the relationship (46), namely,

$$
\begin{gathered}
K=\epsilon_{i} g^{\prime}\left(p_{i}\right)-k T\left[f\left(p_{i}\right)+p_{i} f^{\prime}\left(p_{i}\right)\right] \Rightarrow \\
{\left[f\left(p_{i}\right)+p_{i} f^{\prime}\left(p_{i}\right)\right]-\beta\left[\epsilon_{i} g^{\prime}\left(p_{i}\right)-K\right]=0,} \\
\beta \equiv 1 / k T .
\end{gathered}
$$

\subsection{Tsallis measure with linear constraints}

We have, for any real number $q$ the information measure $(28)$ built up with $(26 ; 33 ; 34)$

$$
f\left(p_{i}\right)=\frac{\left(1-p_{i}^{q-1}\right)}{q-1},
$$

and, in the energy-constraint of Eq. (30)

$$
g\left(p_{i}\right)=p_{i}
$$

so that $f^{\prime}\left(p_{i}\right)=-p_{i}^{q-2}$ and Eq. (64) becomes, with $\beta=(1 / k T)$,

$$
q p_{i}^{q-1}=1+(q-1) \beta K-(q-1) \beta \epsilon_{i},
$$

which after normalization yields a distribution often referred to as the Tsallis' one (33)

$$
\begin{aligned}
p_{i} & =Z_{q}^{-1}\left[1-(q-1) \beta^{\prime} \epsilon_{i}\right]^{1 /(q-1)} \\
Z_{q} & =\sum_{i}\left[1-(q-1) \beta^{\prime} \epsilon_{i}\right]^{1 /(q-1)}
\end{aligned}
$$

where $\beta^{\prime} \equiv \beta /(1+(q-1) \beta K)$.

\subsection{Tsallis measure with non-linear constraints}

The information measure is still the one built up with the function $f\left(p_{i}\right)$ of (65), but we use now the so-called Curado-Tsallis constraints (35) that arise if one uses

$$
U=\langle E\rangle=\sum_{i=1}^{W} g\left(p_{i}\right) \epsilon_{i},
$$

with

Eq. (64) leads to

$$
g\left(p_{i}\right)=p_{i}^{q} \Rightarrow g^{\prime}\left(p_{i}\right)=q p_{i}^{q-1}
$$

$$
p_{i}=\left(\frac{1}{q}\right)^{1 /(q-1)}\left[1-(1-q) \beta \epsilon_{i}\right]^{1 /(1-q),}
$$

and, after normalization, one is led to the Curado-Tsallis distribution (35)

$$
\begin{aligned}
p_{i} & =\left(Z_{q}\right)^{-1}\left[1-(1-q) \beta \epsilon_{i}\right]^{1 /(1-q)} \\
Z_{q} & =\sum_{i}\left[1-(1-q) \beta \epsilon_{i}\right]^{1 /(1-q)}
\end{aligned}
$$




\subsection{Exponential entropic form}

This measure is given in $(36 ; 37)$ and also used in $(38)$. One has

$$
f\left(p_{i}\right)=\frac{1-\exp \left(-b p_{i}\right)}{p_{i}}-S_{0}
$$

where $b$ is a positive constant and $S_{0}=1-\exp (-b)$, together with

$$
g\left(p_{i}\right)=\frac{1-e^{-b p_{i}}}{S_{0}} \Rightarrow g^{\prime}\left(p_{i}\right)=\frac{b e^{-b p_{i}}}{S_{0}}
$$

which, inserted into (64), after a little algebra, leads to

$$
p_{i}=\frac{1}{b}\left[\ln \frac{b}{S_{0}-\beta K}+\ln \left(1-\frac{\beta \epsilon_{i}}{S_{0}}\right)\right] .
$$

which, after normalization, gives the correct answer (37).

\section{Conclusions}

We have seen that the set of equations

$$
\begin{gathered}
\sum_{i}\left[C_{i}^{(1)}+C_{i}^{(2)}\right] d p_{i}=0, \\
C_{i}^{(1)}=\left[\sum_{v=1}^{M} P_{v} a_{i}^{v}+\epsilon_{i}\right] g^{\prime}\left(p_{i}\right) \\
C_{i}^{(2)}=-T \frac{\partial S}{\partial p_{i}}
\end{gathered}
$$

yields a probability distribution that coincides with the PD provided by either

- the MaxEnt's, SM axiomatics of Jaynes'

- our two postulates (15) and (16).

We remind the reader that in our instance the postulates start with

1. the macroscopic thermodynamic relation $d E=T d S+\sum_{v} P_{v} d A_{v}$, adding to it

2. Boltzmann's conjecture of an underlying microscopic scenario ruled by microstate probability distributions.

The two postulates combine then (i) a well-tested macroscopic result with (ii) a by now un uncontestable microscopic state of affairs (which was not the case in Boltzmann's times). Thus we may dare to assert that the two axioms we are here advancing are intuitively intelligible from a physical laboratory standpoint. This cannot be said neither for Gibbs' ensemble nor for Jaynes' extremizing of the Observer's ignorance, their extraordinary success notwithstanding, since they introduce concepts like ensemble or ignorance that are not easily assimilated to laboratory equipment. We must insist: there is nothing wrong with making use of these concepts, of course. We just tried to see whether they could be eliminated from the axioms of the theory.

Summing up, we have revisited the foundations of statistical mechanics and shown that it is possible to reformulate it on the basis of just a basic thermodynamics' relation plus Boltzmann's "atomic" hypothesis. The latter entails (1) the (obvious today, but not in 1866) existence of a microscopic realm ruled by probability distributions. 


\section{Appendix I}

Here we prove that Eqs. (24) are obtained via the MaxEnt variational problem (27). Assume now that you wish to extremize $S$ subject to the constraints of fixed valued for i) $U$ and ii) the $M$ values $A_{v}$. This is achieved via Lagrange multipliers (1) $\beta$ and (2) $M \gamma_{v}$. We need also a normalization Lagrange multiplier $\xi$. Recall that

$$
A_{v}=\left\langle\mathcal{R}_{v}\right\rangle=\sum_{i} p_{i} a_{i}^{v}
$$

with $a_{i}^{v}=\left\langle i\left|\mathcal{R}_{v}\right| i\right\rangle$ the matrix elements in the chosen basis $\langle i\rangle$ of $\mathcal{R}_{v}$. The MaxEnt variational problem becomes now $\left(U=\sum_{i} p_{i} \epsilon_{i}\right)$

$$
\delta_{\left\{p_{i}\right\}}\left[S-\beta U-\sum_{v=1}^{M} \gamma_{v} A_{v}-\xi \sum_{i} p_{i}\right]=0,
$$

leading, with $\gamma_{v}=\beta \lambda_{v}$, to the vanishing of

$$
\delta_{p_{m}} \sum_{i}\left(p_{i} f\left(p_{i}\right)-\left[\beta p_{i}\left(\sum_{v=1}^{M} \lambda_{v} a_{i}^{v}+\epsilon_{i}\right)+\xi p_{i}\right]\right),
$$

so that the 2 quantities below vanish

$$
\begin{gathered}
f\left(p_{i}\right)+p_{i} f^{\prime}\left(p_{i}\right)-\left[\beta\left(\sum_{v=1}^{M} \lambda_{v} a_{i}^{v}+\epsilon_{i}\right)+\xi\right] \\
\Rightarrow \text { if } \xi \equiv \beta K \\
\left.f\left(p_{i}\right)+p_{i} f^{\prime}\left(p_{i}\right)-\beta p_{i}\left(\sum_{v=1}^{M} \lambda_{v} a_{i}^{v}+\epsilon_{i}\right)+K\right] \\
\Rightarrow 0=T_{i}^{(1)}+T_{i}^{(2)} .
\end{gathered}
$$

Clearly, (26) and the last equality of (79) are one and the same equation! Our equivalence is thus proven.

\section{Acknowledgments}

This work is founded by the Spain Ministry of Science and Innovation (Project FIS2008-00781) and by FEDER funds (EU).

\section{References}

[1] R. B. Lindsay and H. Margenau, Foundations of physics, NY, Dover, 1957.

[2] J. Willard Gibbs, Elementary Principles in Statistical Mechanics, New Haven, Yale University Press, 1902.

[3] E.T. Jaynes, Probability Theory: The Logic of Science, Cambridge University Press, Cambridge, 2005.

[4] W.T. Grandy Jr. and P. W. Milonni (Editors), Physics and Probability. Essays in Honor of Edwin T. Jaynes, NY, Cambridge University Press, 1993.

[5] E. T. Jaynes Papers on probability, statistics and statistical physics, edited by R. D. Rosenkrantz, Dordrecht, Reidel, 1987.

[6] E. A. Desloge, Thermal physics NY, Holt, Rhinehart and Winston, 1968.

[7] E. Curado, A. Plastino, Phys. Rev. E 72 (2005) 047103.

[8] A. Plastino, E. Curado, Physica A 365 (2006) 24 
[9] A. Plastino, E. Curado, International Journal of Modern Physics B 21 (2007) 2557

[10] A. Plastino, E. Curado, Physica A 386 (2007) 155

[11] A. Plastino, E. Curado, M. Casas, Entropy A 10 (2008) 124

[12] International Journal of Modern Physics B 22, (2008) 4589

[13] E. Curado, F. Nobre, A. Plastino, Physica A 389 (2010) 970.

[14] The MaxEnt treatment assumes that these macrocopic parameters are the expectation values of appropiate operators.

[15] C. E. Shannon, Bell System Technol. J. 27 (1948) 379-390.

[16] A. Plastino and A. R. Plastino in Condensed Matter Theories, Volume 11, E. Ludeña (Ed.), Nova Science Publishers, p. 341 (1996).

[17] A. Katz, Principles of Statistical Mechanics, The information Theory Approach, San Francisco, Freeman and Co., 1967.

[18] D. J. Scalapino in Physics and probability. Essays in honor of Edwin T. Jaynes edited by W. T. Grandy, Jr. and P. W. Milonni (Cambridge University Press, NY, 1993), and references therein.

[19] T. M. Cover and J. A. Thomas, Elements of information theory, NY, J. Wiley, 1991.

[20] B. Russell, A history of western philosophy (Simon \& Schuster, NY, 1945).

[21] P. W. Bridgman The nature of physical theory (Dover, NY, 1936).

[22] P. Duhem The aim and structure of physical theory (Princeton University Press, Princeton, New Jersey, 1954).

[23] R. B. Lindsay Concepts and methods of theoretical physics (Van Nostrand, NY, 1951).

[24] H. Weyl Philosophy of mathematics and natural science (Princeton University Press, Princeton, New Jersey, 1949).

[25] D. Lindley, Boltzmann's atom, NY, The free press, 2001.

[26] M. Gell-Mann and C. Tsallis, Eds. Nonextensive Entropy: Interdisciplinary applications, Oxford, Oxford University Press, 2004.

[27] G. L. Ferri, S. Martinez, A. Plastino, Journal of Statistical Mechanics, P04009 (2005).

[28] R.K. Pathria, Statistical Mechanics (Pergamon Press, Exeter, 1993).

[29] F. Reif, Statistical and thermal physics (McGraw-Hill, NY, 1965).

[30] J. J.Sakurai, Modern quantum mechanics (Benjamin, Menlo Park, Ca., 1985).

[31] B. H. Lavenda, Statistical Physics (J. Wiley, New York, 1991); B. H. Lavenda, Thermodynamics of Extremes (Albion, West Sussex, 1995).

[32] K. Huang, Statistical Mechanics, 2nd Edition. (J. Wiley, New York, 1987). Pages 7-8.

[33] C. Tsallis, Braz. J. of Phys. 29, 1 (1999); A. Plastino and A. R. Plastino, Braz. J. of Phys. 29, 50 (1999).

[34] A. R. Plastino and A. Plastino, Phys. Lett. A 177, 177 (1993).

[35] E. M. F. Curado and C. Tsallis, J. Phys. A, 24, L69 (1991).

[36] E. M. F. Curado, Braz. J. Phys. 29, 36 (1999).

[37] E. M. F. Curado and F. D. Nobre, Physica A 335, 94 (2004).

[38] N. Canosa and R. Rossignoli, Phys. Rev. Lett. 88, 170401 (2002). 


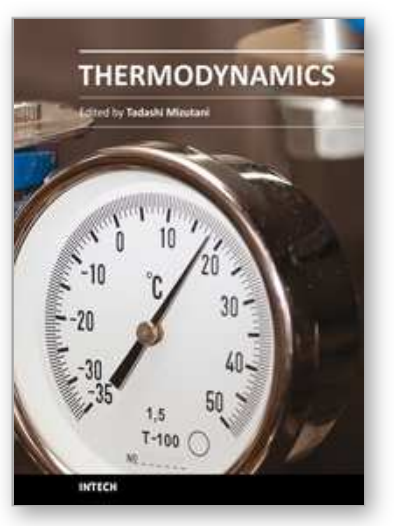

\author{
Thermodynamics \\ Edited by Prof. Mizutani Tadashi
}

ISBN 978-953-307-544-0

Hard cover, 440 pages

Publisher InTech

Published online 14, January, 2011

Published in print edition January, 2011

Progress of thermodynamics has been stimulated by the findings of a variety of fields of science and technology. The principles of thermodynamics are so general that the application is widespread to such fields as solid state physics, chemistry, biology, astronomical science, materials science, and chemical engineering. The contents of this book should be of help to many scientists and engineers.

\title{
How to reference
}

In order to correctly reference this scholarly work, feel free to copy and paste the following:

A. Plastino and M. Casas (2011). New Microscopic Connections of Thermodynamics, Thermodynamics, Prof. Mizutani Tadashi (Ed.), ISBN: 978-953-307-544-0, InTech, Available from:

http://www.intechopen.com/books/thermodynamics/new-microscopic-connections-of-thermodynamics

\section{INTECH}

open science | open minds

\section{InTech Europe}

University Campus STeP Ri

Slavka Krautzeka 83/A

51000 Rijeka, Croatia

Phone: +385 (51) 770447

Fax: +385 (51) 686166

www.intechopen.com

\section{InTech China}

Unit 405, Office Block, Hotel Equatorial Shanghai

No.65, Yan An Road (West), Shanghai, 200040, China

中国上海市延安西路65号上海国际贵都大饭店办公楼405单元

Phone: +86-21-62489820

Fax: $+86-21-62489821$ 
(C) 2011 The Author(s). Licensee IntechOpen. This chapter is distributed under the terms of the Creative Commons Attribution-NonCommercialShareAlike-3.0 License, which permits use, distribution and reproduction for non-commercial purposes, provided the original is properly cited and derivative works building on this content are distributed under the same license. 